\title{
The Oncotype DX recurrence score impact on the management of ER-positive, HER2-negative, node-negative breast cancer
}

\section{Corresponding author:}

Abdalla Saad Abdalla Al-Zawi, Basildon \& Thurrock University Hospital, Nethermayne, Basildon, Essex, United Kingdom,

e-mail: abdalasaad@gmail.com
Medical Research Journal 2021; Volume 6, Number 3, 211-216 10.5603/MRJ.a2021.0041 Copyright (C) 2021 Via Medica ISSN 2451-2591 e-ISSN 2451-4101

\begin{abstract}
Introduction: Oncotype DX recurrence score is used to categorize estrogen-receptor-positive, human epidermal growth factor receptor-2 negative, lymph-node negative early breast cancer in high- or low-recurrence risk groups. It has a guiding significance for whether post-operative chemotherapy or only hormonal manipulation is selected as an adjuvant treatment.

Aims: Assess the impact of the Oncotype DX recurrence score on adjuvant chemotherapy-related management decision-making in the cases of ER+ve, HER2-ve, LN-ve early breast cancer in our local unit. Material and methods: A cohort of 76 patients with early breast cancer were included, two had bilateral disease. All were operated for estrogen-receptor-positive, human epidermal growth factor receptor-2 negative, lymph-node negative early breast cancer. Tumor grade, Ki67 proliferative index, PREDICT and Oncotype DX recurrence score results were obtained in addition to the offered treatment information for each case. Results: After the primary tumor surgery and an Oncotype DX recurrence score assessment, 18 patients (24\%) were eligible for adjuvant chemotherapy; out of them, 10 patients $(56 \%)$ had a chemotherapy absolute survival benefit of $>15 \%$ at 10 years, where 5 patients $(30 \%)$ had a chemotherapy relative survival benefit of $\sim 6.5 \%, 3$ patients (17\%) had a chemotherapy relative survival benefit of $\sim 1.6 \%$. In this cohort, 10 patients (13\%) had a low Oncotype DX recurrence score; however, they received adjuvant chemotherapy based on other clinico-biological parameters. The other 48 patients (63\%) with a low recurrence risk were spared potential adverse events related to the systemic therapy. Based on the menopausal status, every patient had received suitable hormonal manipulation therapy. The data also revealed the absence of a relationship between the Ki67 proliferative index and the Oncotype DX recurrence score $(p=0.06)$; moreover, the size of the tumor did not correlate with the Oncotype DX recurrence score $(p=0.5)$.

Conclusion: The Oncotype DX recurrence score provides a credible prediction of distant disease recurrence risk in early breast cancer; however, it does not correlate with other prognostic markers, such as the Ki67 proliferative index as well as the tumor size. In this cohort, the use of the Oncotype DX recurrence score led to a $24 \%$ rate of treatment recommendations in the direction of adjuvant chemotherapy in addition to anti-hormonal therapy for estrogen-receptor-positive, human epidermal growth factor receptor-2 negative, lymph-node negative early breast cancer.
\end{abstract}

Key words: breast cancer, Oncotype DX, estrogen receptor, Ki67, HER2, chemotherapy

Med Res J 2021; 6 (3): 211-216

\section{Introduction}

Worldwide, breast cancer is regarded as the most frequent malignancy; however, it occupies the fifth place on the list of cancer-related mortality after lung, colon, liver, and stomach cancers. According to the 2017 UK data, in both genders, it is reported to be the most diagnosed cancer with over 55,000 new cases diagnosed yearly. It accounts for $15 \%$ of all newly diagnosed cancers and also is considered to be the most 
common female cancer. Up to $80 \%$ of these cases are categorized as early breast cancer $[1,2]$.

The standard treatment of estrogen-receptor-positive $(E R+v e)$, human epidermal growth factor receptor-2 negative (HER2-ve), lymph-node negative ( $\mathrm{LN}-\mathrm{ve}$ ) early breast cancer is hormonal manipulation therapy administered in addition to chemotherapy and radiotherapy as appropriate. Adjuvant-chemotherapy treatment decision in breast cancer may be affected by different biological and molecular parameters, such as tumor size, nodal status, histological grade, oestrogen hormone status, the HER2 receptor expression or the Ki67 proliferative index (Ki67-PI) (Fig. 1). The systemic treatment recommendation is also supported by online tools, such as PREDICT and NPI (Nottingham Prognostic Index) - these tools utilize demographic patient characteristics as well as tumor's biological markers [3]. Few molecular technologies have been developed to aid in decision-making when dealing with moderately aggressive tumors, and the cancer-biology characteristics do not provide enough justification to proceed with adjuvant chemotherapy. Such technologies include the Oncotypoe DX recurrence score (RS) and Mammaprint70 [4]. The RS is an RT-PCR (real-time reverse transcriptase-polymerase chain reaction) based multigene assay, involving 21 genes, performed on fixed paraffin-embedded tissues of the excised tumors. Its result is used for the prediction of adjuvant chemotherapy benefits and disease prognosis.

\section{Material and methods}

The patients that were enrolled in this study, according to the inclusion criteria for had (1) operable estrogen-receptor positive, human epidermal growth factor receptor-2 negative and lymph-node negative (including micro-metastatic disease) operable early breast cancer, (2) an intermediate risk of distant recurrence using a validated tool such as PREDICT or the NPI (Nottingham Prognostic Index). A cohort of 76 patients with operable early breast cancer was included; two of them had bilateral disease, and the RS was examined for 78 tumors. The patients' age ranged from 37 to 74 years, with a median age of 55 (Fig. 2). All the patients underwent tumor surgical excision with breast conservation surgery or mastectomy in addition to axillary node staging via sentinel lymph node biopsy or axillary node sampling. Tumor size, grade, Ki67-PI, PREDICT, and Oncotype DX recurrence score results, and the information related to the offered treatment was obtained and analyzed. The value of $20 \%$ was used as a cut-off point for the Ki67-PI level, a low Ki67-PI is $<20 \%$, whereas it is high if the level is $\geq 20 \%$.

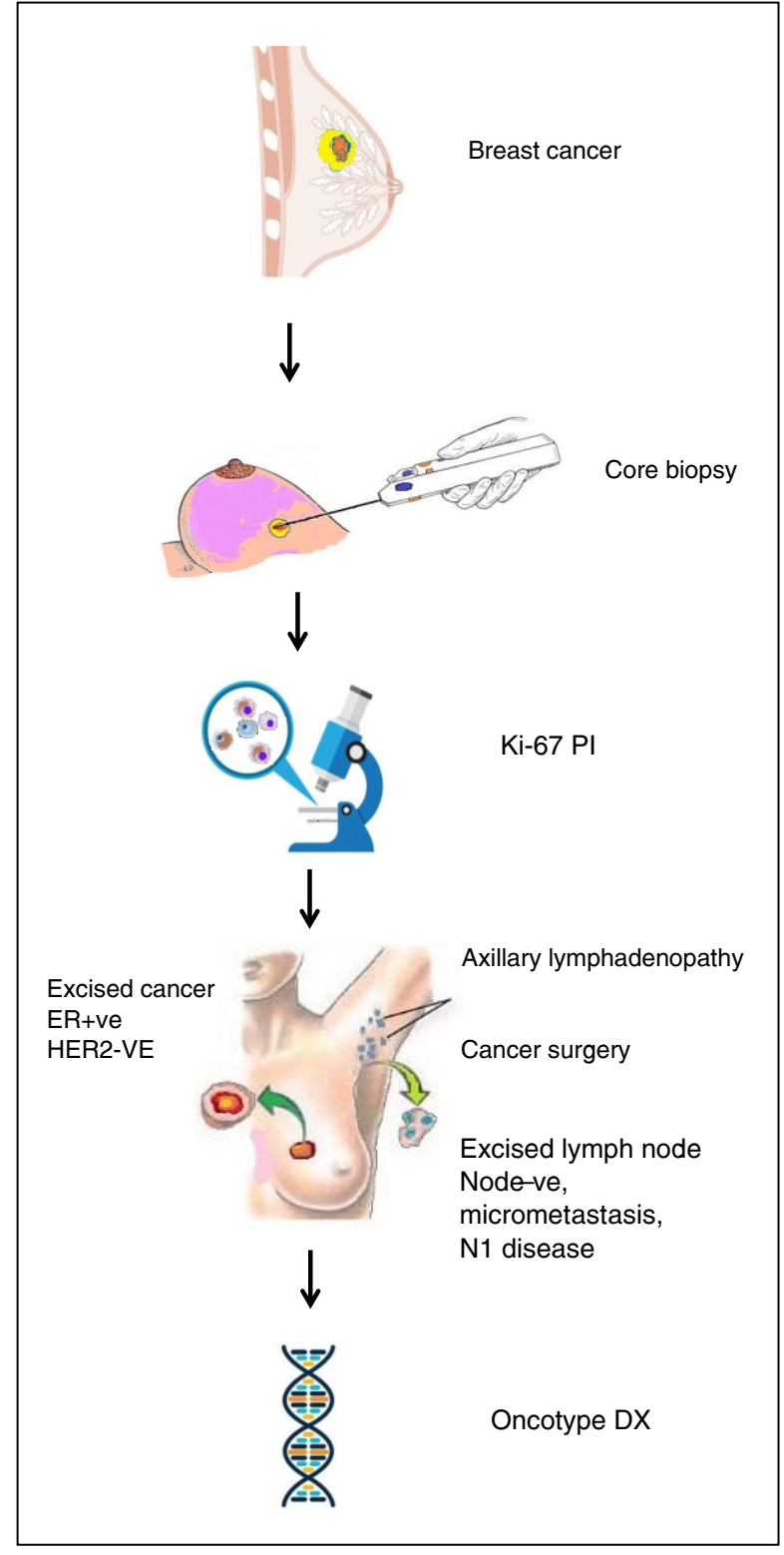

Figure 1. Oncotype DX and Ki67-PI in breast cancer diagnosis workup (the illustration was created by $\mathrm{Dr}$. Abdalla Saad Abdalla Al-Zawi)

\section{Results}

The Ki67-PI analysis categorized most of the cohort within the low Ki67-PI group < 20\% (68\%), where only 25 tumors showed high Ki67-PI expression $\geq 20 \%$ (32\%). There were only 17 (22\%) tumors with a high Ki67-PI and a low Oncotype DX recurrence score; on the contrary, 8 patients had a low Ki67-PI with a high RS (Tab. 1). Our data revealed the absence of a relationship between the Ki67-PI and the RS $(p=0.06)$. Looking at the tumor size, T3 tumors (>50mm) were detected in 5 lesions (6\%); 37 of them had a low RS. However, 53 lesions (68\%) 


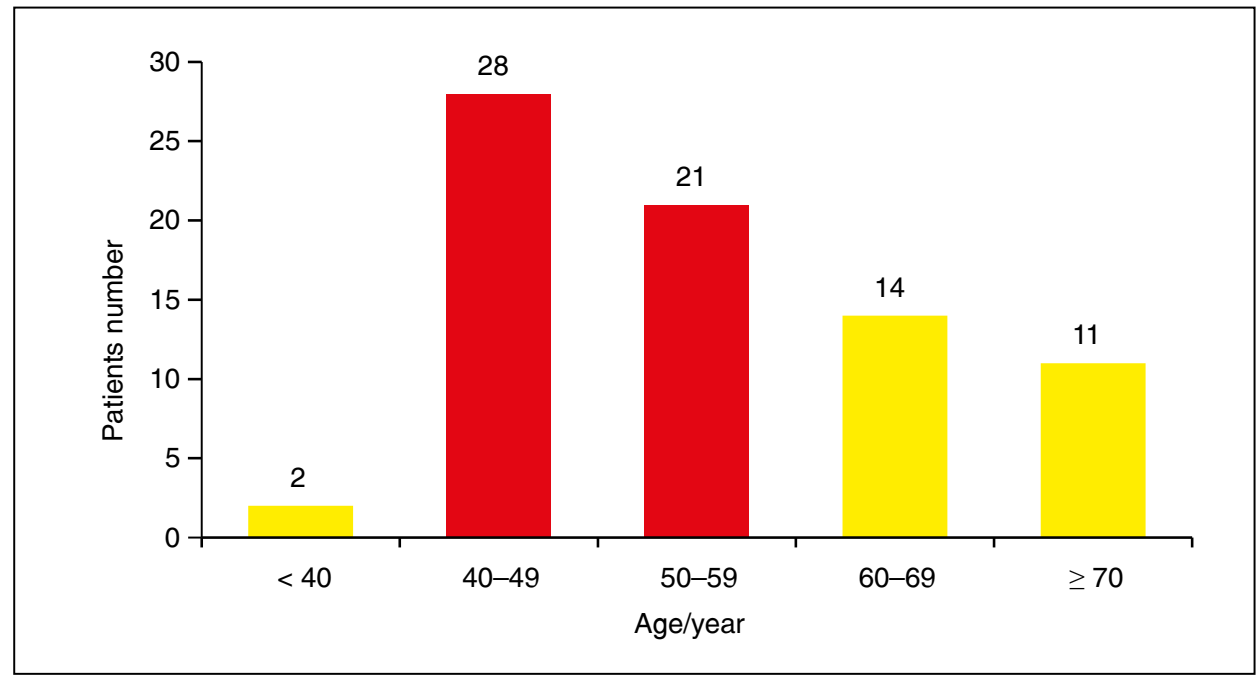

Figure 2. Age distribution of 76 patients treated for early breast cancer

Table 1. The Oncotype DX recurrence score and the Ki67 proliferative index in 78 excised tumors Age group $\leq \mathbf{5 0}$ years

Oncotype DX recurrence score Chemotherapy (CTH) benefit Low Ki67-PI group* High Ki67-PI group**

\begin{tabular}{|c|c|c|c|}
\hline $0-15$ & No CTH benefit & 12 & 6 \\
\hline $16-20$ & 1.6\% CTH benefit & 2 & 4 \\
\hline $21-25$ & $\sim 6.5 \% \mathrm{CTH}$ benefit & 4 & 2 \\
\hline $26-100$ & $15 \%$ absolute CTH benefit & 1 & 3 \\
\hline \multicolumn{4}{|c|}{ Age group > 50 years } \\
\hline $0-25$ & No CTH benefit & 24 & 13 \\
\hline $26-100$ & $15 \%$ absolute benefit & 2 & 5 \\
\hline
\end{tabular}

*Low Ki67-PI < 20\%; **High Ki67-PI $\geq 20 \%$

were T2 tumors (20-50 mm). Out of these, $37(70 \%)$ had a low RS, and only $8(15 \%)$ showed a high $R S(p=0.5)$. This means that tumor size is not associated with an Oncotype DX recurrence score. Regarding tumor grade, G2 lesions were seen in 59 tumors (76\%), only 5 (6\%) were associated with RS > 20. Sixteen lesions (21\%) were G3 tumors, only $3(4 \%)$ had a high RS $(p=0.46)$.

By using the PREDICT online tool, two (3\%) patients in the age group of $\leq 50$ years had a high PREDICT and RS; one patient aged $\leq 50$ had a low PREDICT score and a high RS. In the age group $>50$ years, 4 patients (5\%) had a high PREIDCT with a low RS, 4 patients (5\%) a low PREDICT and RS; only only 2 had high both the PREDICT and the RS. After primary tumor surgery and an RS assessment, in total, 28 patients (36\%) were eligible for adjuvant chemotherapy (Tab. 2). Adjuvant chemotherapy was determined by RS in 18 patients $(24 \%)$. Out of them, ten patients $(56 \%)$ had a chemotherapy absolute survival benefit of $>15 \%$ at
10 years, where $5(30 \%)$ patients had a chemotherapy relative survival benefit of $\sim 6.5 \%, 3$ patients (17 \%) patients had a chemotherapy relative survival benefit of $\sim 1.6 \%$. Ten patients (13\%) had a low RS; however, they had chemotherapy based on other clinico-pathological parameters. This means 48 patients (63\%) with a low recurrence risk had been spared potential adverse events related to the systemic therapy.

\section{Discussion}

The Oncotype DX recurrence score is a 21-multigene real-time reverse transcription polymerase chain reaction assay (Fig. 3). It is performed on the excised tumor cells, and the outcomes are presented as numerical results $(0-100 \%)$ (Tab. 3). It is recommended to be used as a systemic recurrence predictive tool for guiding adjuvant-chemotherapy decisions in 
Table 2. The Oncotype DX recurrence score and chemotherapy indication for the cohort

\begin{tabular}{|c|c|c|c|}
\hline \multicolumn{4}{|c|}{ Age group $\leq \mathbf{5 0}$ years } \\
\hline $\begin{array}{l}\text { Oncotype DX } \\
\text { recurrence score }\end{array}$ & Chemotherapy (CTH) benefit & Total patients number (\%) & Patients for CTH(\%) \\
\hline $0-15$ & No CTH benefit & $17(22)$ & $4(5)$ \\
\hline $16-20$ & $\sim 1.6 \%$ CTH benefit & $6(8)$ & $3(4)$ \\
\hline $21-25$ & $\sim 6.5 \% \mathrm{CTH}$ benefit & $7(9)$ & $5(7)$ \\
\hline $26-100$ & 15\% absolute CTH benefit & $3(4)$ & $3(4)$ \\
\hline \multicolumn{4}{|c|}{ Age group $>50$ years } \\
\hline $0-25$ & No CTH benefit & $36(47)$ & $6(8)$ \\
\hline $26-100$ & 15\% absolute CTH benefit & $7(9)$ & $7(9)$ \\
\hline
\end{tabular}

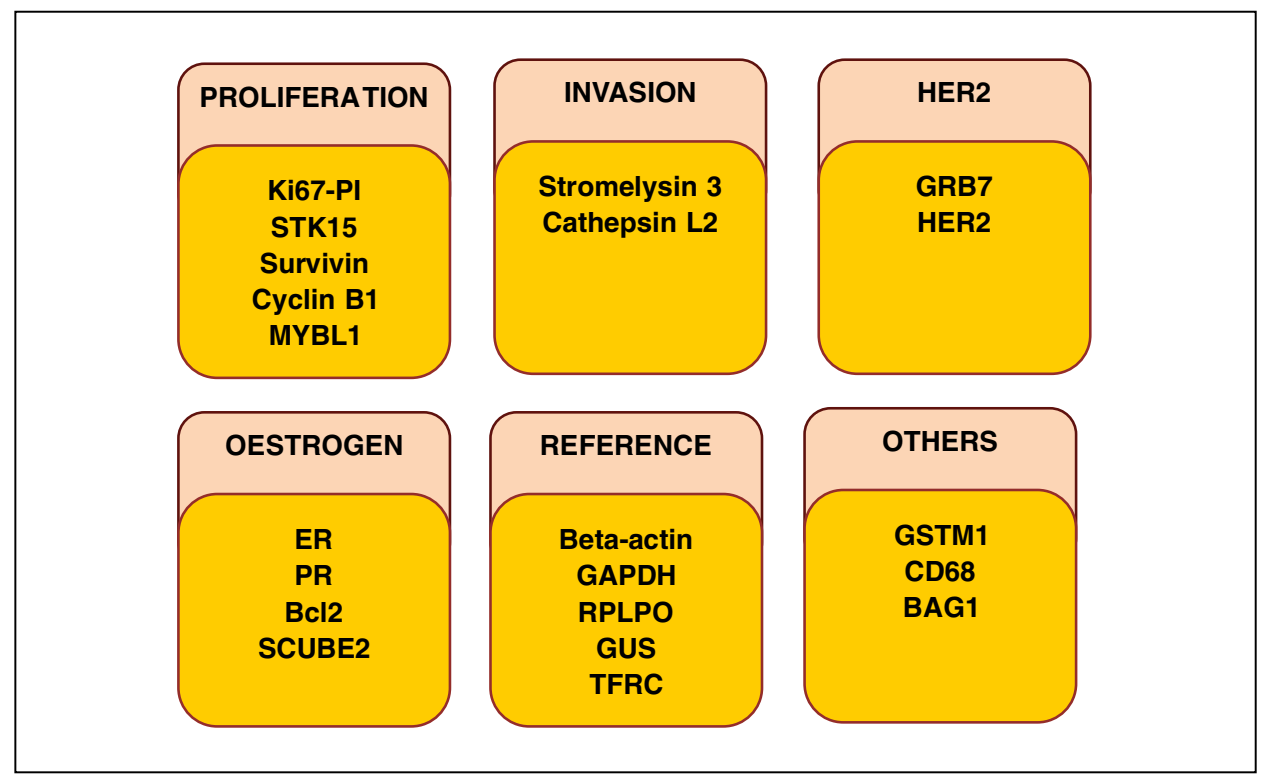

Figure 3. The 21 genes tested by RT-PCR (real-time reverse transcriptase-polymerase chain reaction) to generate the Oncotype DX recurrence score

moderately aggressive ER+ve, HER2-ve, LN-ve early breast cancers [5]. This gene panel is formed of five reference genes and sixteen cancer-related genes, the latter include those associated with tumor invasion, cell proliferation, and hormone receptors expression. The test result will have a recurrence score between 0 and 100. This score will give an idea about the likelihood of disease recurrence within 10 years after diagnosis [6], and it estimates the chemotherapeutic benefit in ER+ve, HER2-ve, LN-ve early breast cancer [7]. After the introduction of the Oncotype DX recurrence score used to measure the systemic recurrence score risk on the basis of tumor genomic signature, the adjuvant-chemotherapy indications in ER+ve, HER2-ve, LN-ve breast cancer disease have been changed notably. The genomic assay results (the Oncotype DX recurrence score) are used as a decision-making tool and to aid in determining whether the patient will be only treated with adjuvant hormonal therapy (a low RS) or, additionally, with adjuvant chemotherapy (a high RS), so that overtreatment can be avoided in the earlier group. The Oncotype DX recurrence score also has a place in male breast cancer management [8] because male breast cancer, compared with female breast cancer, is usually diagnosed at a later stage, has a larger tumor size, more lymph node-positive disease, higher rates of estrogen-receptor positivity and lower rates of HER2 expression.

The Oncotype DX recurrence score results interpretation is categorized according to age (Tab. 3), for patients $>50$ years of age, it is classified into two groups:

(I) Oncotype DX recurrence score of 0-25: There is a low risk of recurrence and benefits of adjuvant chemotherapy are not likely to outweigh the risks of side effects. 
Table 3. Interpretation of Oncotype DX recurrence score and the chemotherapy survival benefit at 10 years

\begin{tabular}{lc}
\hline $\begin{array}{l}\text { Age group } \leq \mathbf{5 0} \text { years } \\
\begin{array}{l}\text { Oncotype } \mathbf{D X} \text { recurrence } \\
\text { score }\end{array}\end{array}$ & $\begin{array}{c}\text { Chemotherapy (CTH) } \\
\text { benefit }\end{array}$ \\
\hline $0-15$ & No CTH benefit \\
$16-20$ & $\sim 1.6 \%$ CTH benefit \\
$21-25$ & $\sim 6.5 \%$ CTH benefit \\
$26-100$ & $15 \%$ absolute CTH benefit \\
\hline Age group $>\mathbf{5 0}$ years & No CTH benefit \\
\hline $0-25$ & $15 \%$ absolute CTH benefit \\
$26-100$ &
\end{tabular}

(II) Oncotype DX recurrence score of 26-100: Breast cancer is associated with a high risk of recurrence, so adjuvant chemotherapy benefits are likely to be greater than the risks of side effects.

For patients aged 50 years and younger, the Oncotype DX recurrence score results interpretation is categorized into four groups (Tab. 3 ):

(I) Oncotype DX recurrence score of 0-15: The cancer is associated with a low recurrence risk, and chemotherapy benefits are not likely to outweigh the risks of chemotherapy adverse effects.

(II) Oncotype DX recurrence score of 16-20: Breast cancer possesses a low to medium recurrence risk, and chemotherapy benefits are not likely to outweigh the risks of chemotherapy adverse effects.

(III) Oncotype DX recurrence score of 21-25: Breast cancer has a medium recurrence risk, and chemotherapy benefits are likely to be greater than the risks of side effects.

(IV) Oncotype DX recurrence score of 26-100: Breast cancer is associated with a high recurrence risk, and chemotherapy benefits are likely to be greater than the risks of adverse effects [9].

A number of studies have, indeed, reported different rates of treatment recommendations supporting adjuvant chemotherapy in ER+ve, HER2-ve, and LN-ve early breast cancer. In TAILORx (Trial Assigning Individualized Options for Treatment) phase III clinical trial, it was reported that adjuvant chemotherapy can be omitted for ER+ve, HER2-ve, and LN-ve breast cancer patients aged $\leq 50$ years with the Oncotype DX recurrence score $\leq 15$, in addition to patients aged $>50$ years and $R S \leq 25$ [9]. In 2019, Dieci et al. [5] published the ROXANE Italian prospective study, which evaluated the impact of the Oncotype DX recurrence score on adjuvant treatment decision for patients with early breast cancer; 251 patients were included in the study. The authors found that $15 \%$ of the patients had a post-RS test recommendation to chemotherapy and hormonal manipulation. Overall, the change in management recommendation from pre-RS testing to post-RS testing occurred in $30 \%$ of patients, most frequently from hormonal treatment and chemotherapy to hormonal therapy alone [5]. In other reports, the rates ranged from 13 to $28 \%$, in our cohort the rate was $24 \%$, which is within the range of the published data [10-13]. The Southwest Oncology Group in phase III of SWOG-8814 trial investigated whether the RS was prognostic in breast-cancer patients treated with tamoxifen alone and whether the RS identified those who might not benefit from anthracycline-based chemotherapy despite higher recurrence risks. The full title of this trial is "Tamoxifen with or without Combination Chemotherapy in Postmenopausal Women who have Undergone Surgery for Breast Cancer". The study showed that adjuvant chemotherapy with cyclophosphamide, doxorubicin, and fluorouracil (CAF) prior to hormonal manipulation of tamoxifen (CAF-T) added survival benefit to the treatment option with tamoxifen alone [14]. This trial was followed by a retrospective study done by Albain et al. [15] in 2010, which assessed the impact of the Oncotype DX recurrence score on disease-free survival by treatment option group (tamoxifen only vs CAF-T). It was found that the Oncotype DX recurrence score is prognostic for node-positive disease treated with tamoxifen alone as there was no benefit of CAF in the cases with a low recurrence score. The high Oncotype DX recurrence score predicts the significant benefit of CAF in breast cancer, associated with an improvement in disease-free survival for patients belonging to this group. A low Oncotype DX recurrence score identifies patients who might not benefit from anthracycline-based chemotherapy, despite the presence of nodal disease [15].

In 2010, Dowsett et al. [16] found in a cohort of 1231 patients that the Oncotype DX recurrence score assessment is an independent predictor of distant disease recurrence in node-negative and node-positive, hormone-receptor-positive breast cancer patients treated with aromatase inhibitors (Al), adding value to estimates with standard clinico-pathologic indicators. Generally speaking, compared with clinical and other biomarkers, the Oncotype DX recurrence score is a more reliable indicator in predicting disease recurrence as well as distant metastasis risk in early ER+ve, HER2-ve, and LN-ve early breast cancer patients. In the patient group associated with a high-risk RS, the additional chemotherapy benefit when combined with hormonal therapy is higher compared to hormonal manipulation alone, where the benefit is minimal in the low-risk group. 


\section{Conclusion}

The Oncotype DX recurrence score use is an effective move toward precision medicine, it allows picking up the group of patients with unfavorable tumor biology to receive the systemic treatment, where a significant number of patients will be spared unnecessary overtreatment. In this cohort, the use of RS testing led to a $24 \%$ rate of recommendations to administer adjuvant chemotherapy for ER+ve, HER2-ve and LN-ve early breast cancer.

\section{References}

1. Khan MA, Henderson L, Clarke D, et al. The Warwick experience of the Oncotype DX $®$ Breast Recurrence Score $\AA$ assay as a predictor of chemotherapy administration. Breast Care (Basel). 2018; 13(5) 369-372, doi: 10.1159/000489131, indexed in Pubmed: 30498424

2. Al-Zawi A. Ki -67 proliferative index as a predictive tool for axillary pathological complete response in node-positive breast cancer International Journal Medical Science. 2020; 7(11): 1-4, doi 10.14445/23939117/ijms-v7i11p101.

3. Tan QX, Qin QH, Yang WP, et al. Prognostic value of Ki67 expression in HR-negative breast cancer before and after neoadjuvant chemotherapy. Int J Clin Exp Pathol. 2014; 7(10): 6862-6870, indexed in Pubmed: 25400769.

4. Siegelmann-Danieli N, Silverman B, Zick A, et al. The impact of the Oncotype DX Recurrence Score on treatment decisions and clinical outcomes in patients with early breast cancer: the Maccabi Healthcare Services experience with a unified testing policy. Ecancermedicalscience. 2013; 7: 380, doi: 10.3332/ecancer.2013.380, indexed in Pubmed: 24386009.

5. Dieci MV, Guarneri V, Zustovich F, et al. Veneto Oncology Network Impact of 21-gene breast cancer assay on treatment decision for patients with T1-T3, N0-N1, estrogen receptor-positive/human epidermal growth receptor 2-negative breast cancer: final results of the prospective multicenter ROXANE study. Oncologist. 2019; 24(11): 1424-1431, doi: 10.1634/theoncologist.2019-0103, indexed in Pubmed: 31152079.

6. McVeigh TP, Hughes LM, Miller N, et al. The impact of Oncotype $\mathrm{DX}$ testing on breast cancer management and chemotherapy prescribing patterns in a tertiary referral centre. Eur J Cancer. 2014;
50(16): 2763-2770, doi: 10.1016/j.ejca.2014.08.002, indexed in Pubmed: 25240289

7. Kim HJ, Choi WJ, Kim HH, et al. Association between Oncotype DX recurrence score and dynamic contrast-enhanced MRI features in patients with estrogen receptor-positive HER2-negative invasive breast cancer. Clin Imaging. 2021; 75: 131-137, doi: 10.1016/j.clinimag.2021.01.021, indexed in Pubmed: 33548871.

8. Zheng A, Zhang L, Ji Z, et al. Oncotype DX for comprehensive treatment in male breast cancer: a case report and literature review. Am J Mens Health. 2019; 13(3): 1557988319847856, doi: 10.1177/1557988319847856, indexed in Pubmed: 31068065

9. Sparano JA, Gray RJ, Makower DF, et al. Adjuvant chemotherapy guided by a 21-gene expression assay in breast cancer. $N$ Engl J Med. 2018; 379(2): 111-121, doi: 10.1056/NEJMoa1804710, indexed in Pubmed: 29860917.

10. Rabie MA, Rankin A, Burger A, et al. The effect of Oncotype DX on adjuvant chemotherapy treatment decisions in early breast cancer. Ann R Coll Surg Engl. 2019; 101(8): 596-601, doi: 10.1308/rcsann.2019.0096, indexed in Pubmed: 31219316.

11. Loncaster J, Armstrong A, Howell S, et al. Impact of Oncotype DX breast Recurrence Score testing on adjuvant chemotherapy use in early breast cancer: Real world experience in Greater Manchester, UK. Eur J Surg Oncol. 2017; 43(5): 931-937, doi: 10.1016/j.ejso.2016.12.010, indexed in Pubmed: 28111076.

12. Crolley VE, Marashi H, Rawther S, et al. The impact of Oncotype DX breast cancer assay results on clinical practice: a UK experience. Breast Cancer Res Treat. 2020; 180(3): 809-817, doi: 10.1007/s10549020-05578-6, indexed in Pubmed: 32170635

13. Chang $\mathrm{CH}$, Lin $\mathrm{YH}$. Using Oncotype DX as an additional treatment decision tool in early breast cancer: a retrospective analysis from a single institution in Taiwan. Ther Radiol Oncol. 2018; 2: 7, doi: 10.21037/tro.2018.01.06

14. Albain KS, Barlow WE, Ravdin PM, et al. Breast Cancer Intergroup of North America. Adjuvant chemotherapy and timing of tamoxifen in postmenopausal patients with endocrine-responsive, node-positive breast cancer: a phase 3, open-label, randomised controlled trial. Lancet. 2009; 374(9707): 2055-2063, doi: 10.1016/S0140-6736(09)61523-3, indexed in Pubmed: 20004966.

15. Albain KS, Barlow WE, Shak S, et al. Breast Cancer Intergroup of North America. Prognostic and predictive value of the 21-gene recurrence score assay in postmenopausal women with node-positive, oestrogen-receptor-positive breast cancer on chemotherapy: a retrospective analysis of a randomised trial. Lancet Oncol. 2010; 11(1): 55-65, doi: 10.1016/S1470-2045(09)70314-6, indexed in Pubmed: 20005174.

16. Dowsett $M$, Cuzick J, Wale $C$, et al. Prediction of risk of distant recurrence using the 21-gene recurrence score in node-negative and node-positive postmenopausal patients with breast cancer treated with anastrozole or tamoxifen: a TransATAC study. J Clin Oncol. 2010; 28(11): 1829-1834, doi: 10.1200/JCO.2009.24.4798, indexed in Pubmed: 20212256. 